DOI: https://doi.org/10.11144/Javeriana.upsy17-3.gvdi

\title{
Graves vulneraciones de derechos en la infancia y adolescencia: variables de funcionamiento familiar*
}

\section{Serious Violations of Rights in Children and Adolescents: Variables of Family Functioning}

Recepción: 14 Agosto 2015 | Aceptación: 19 Agosto 2017

Lorena Contreras Taibo

Universidad Diego Portales, Chile

ORCID: http://orcid.org/0000-0002-3406-768X

Catalina Paulsen Gutiérrez

Universidad Diego Portales, Chile

Esteban Gómez Muzzio

Fundación América por la Infancia, Chile

\footnotetext{
a Autor de correspondencia. Correo

electrónico:lorena.contreras@udp.cl
}

Para citar este artículo: Contreras Taibo, L., Paulsen Gutiérrez, C., \&Gómez Muzzio, E. (2018). Graves vulneraciones de derechos en la infancia y adolescencia: variables de funcionamiento familiar. Universitas Psychologica, 17(3), 1-13. https://doi.org/ 10.11144/Javeriana.upsy17-3.gvdi

\section{RESUMEN}

La presente investigación tiene por objetivo determinar las características y problemáticas que se presentan en el funcionamiento familiar de 389 niños, niñas y adolescentes víctimas de graves vulneraciones de derechos en Chile, mediante la aplicación de la Escala de Evaluación Familiar de Carolina del Norte (NCFAS-G). Sus resultados identifican problemáticas centrales en esta muestra, tales como una moderada vulnerabilidad socioeconómica, dificultades en las interacciones entre sus miembros y con su entorno (familia, vecindario), así como debilitamiento de las competencias parentales. Se concluye que las variables de dinámica relacional de la familia y la pareja, los vínculos afectivos, la parentalidad y la autonomía familiar requieren ser abordadas mediante una intervención integral, sistémica, coordinada y oportuna.

\section{Palabras clave}

vulneración de derechos; maltrato infantil; infancia; relaciones familiares; conducta de apego.

\section{ABSTRACT}

The present research aims to determine the characteristics and problems that occur in the family functioning of 389 children and adolescents victims of serious violations of rights in Chile, through the implementation of the North Carolina Family Assessment Scale (NCFAS-G). The results identify central problems in this sample, such as a moderate socioeconomic vulnerability, difficulties in the interactions between their members and their environment (family, neighbourhood), as well as weakening of parental competences. The conclusion is that the variables of relational dynamics of the family and the couple, affective bonds, parenthood and family autonomy need to be addressed through an integral, systemic, coordinated and timely intervention.

Keywords

rights violation; child abuse; childhood; family relations; attachment behaviour. 
La vulneración de derechos hacia niños/as y adolescentes constituye un grave problema a escala mundial dada su elevada prevalencia, así como a los serios daños a corto y largo plazo que genera en el desarrollo infantil, consecuencias que pueden ser devastadoras. Así, cifras aportadas por el Fondo de las Naciones Unidas para la Infancia - Unicef (Madrid, 2015) señalan que en Chile un $62.2 \%$ de los niños/as ha vivido situaciones de violencia psicológica en su vida, un $50.4 \%$, violencia física leve y un 28.2 $\%$, violencia física grave. Estas y otras situaciones de vulneración de derechos, se asocian a efectos nocivos en el desarrollo cognitivo, emocional, social, sexual y reproductivo de quien las padece (Dubowitz \& Bennet, 2007; Nemeroff, 2016).

Sobre el particular, hoy en día existe un consenso bastante generalizado respecto a la importancia de trabajar no solo con niños y niñas, sino también con las familias en situaciones de vulneración de derechos de sus hijos (Rodrigo, Máiquez, Martín, Byrne \& Rodríguez, 2015), trabajo que tiene por objetivo la restitución del ejercicio de aquellos derechos vulnerados. Esto, mediante la interrupción de dicha vulneración, así como del fortalecimiento del entorno familiar y las competencias de los adultos para proteger el pleno desarrollo del niño/a o adolescente (Arruabarrena, 2001).

Autores como Canales, Flores y Raurich (2014) sostienen que el vínculo constituye el eje articulador central, tanto del propio sujeto como del contexto familiar en el cual se desarrolla. Por tanto, el énfasis de la intervención con familia, y particularmente en contextos de vulneración, apunta justamente al fortalecimiento de esta vinculación de los padres con los hijos, de modo que estos puedan ejercer la parentalidad con mayor eficacia (Rodrigo et al., 2015). En este sentido, podemos entender por vínculo el lazo relacional recíproco que teje la trama social, uniendo a los individuos entre sí y vinculando a los sujetos con los sistemas a los cuales pertenecen. De este modo, instalar o fortalecer este tipo de vinculación bien tratante constituye el mejor "antídoto" frente a la vulneración de derechos.
La relación establecida entre los niños, niñas y adolescentes y sus familias no puede ser comprendida al margen de su contexto, es decir del "entorno físico, social y cultural que influye y contiene (enmarca) a las personas que están en una relación" (Canales et al., 2014, p.9), así como de la trama histórica en la cual esta se inscribe. En esta tarea, se ha adoptado la perspectiva ecológica o ecosistémica desarrollada por Bronfenbrenner (1987) y actualizada por Bronfenbrenner y Evans (2000) y Bronfenbrenner y Morris (2006).

Este enfoque permite disponer de un marco comprehensivo, desde el cual se integran diversas variables que contribuyen a explicar el vínculo y las relaciones familiares y su efecto sobre el desarrollo infanto-juvenil, entendiéndolos como fenómenos situados contextualmente en sistemas que se acoplan. Así, la capacidad de la familia para funcionar eficazmente como marco de desarrollo humano para niños, niñas y jóvenes, depende de la relación que se establezca entre esta y los sucesivos niveles ecológicos que la contienen. La perspectiva ecológica ha sido aplicada por Belsky (1993) y Belsky, Bakermans-Kranenburgy van IJzendoorn (2007) para comprender la etiología del maltrato y negligencia en la infancia y adolescencia, en tanto se ven influidas por "factores que operan en múltiples niveles de análisis (del desarrollo, situacionales, demográficos, histórico-culturales, evolutivos)" (Belsky, 1993, p. 427). Así, la relación que los padres establecen con sus hijos, no solo dependerá de lo que ellos son capaces de ofrecer, sino también de los recursos que posean y las herramientas y servicios que la sociedad pone a disposición. No solo existen factores económicos, sino además culturales y sociales que fomentan y fortalecen contextos favorables para el bienestar infantil (Barudy \& Dantagnan, 2005, 2011; Rodrigo et al., 2015).

En los distintos niveles ecológicos pueden existir factores de riesgo para el ejercicio de la parentalidad, es decir, condiciones biológicas, psicológicas o sociales que incrementan la probabilidad de aparición de un problema determinado (Ayoub et al., 2009; Rose, Holmbeck, Coakley, \& Franks, 2004). Entre 
estos factores, se cuentan la pobreza crónica y el desempleo, las madres que poseen baja escolaridad, la depresión materna, la violencia en la pareja parental, los empleos con horarios extensos y el hacinamiento, entre otros (Evans, Li, \& Whipple, 2013). No obstante, estos factores de riesgo pueden ser contrapesados por factores de protección, los cuales modifican la respuesta de una persona ante un riesgo en un sentido más favorable (Ayoub et al., 2009; Gómez \& Kotliarenco, 2010; Rodrigo, Máiquez, \& Martín, 2010).

Promoviendo el establecimiento de vinculaciones bien tratantes en los diferentes niveles ecológicos en los cuales se desarrollan los niños/as y adolescentes y sus familias, es posible aspirar a la restitución de derechos vulnerados, no solo en ellos, sino también en sus figuras vinculares.

En este marco, el Programa de Familia de la Fundación San Carlos de Maipo, destinado a fortalecer la intervención familiar con niños y niñas vulnerados en sus derechos, impulsó el "Estudio, evaluación y sistematización de los proyectos de intervención focalizados en familias con niños, niñas y jóvenes vulnerados en sus derechos", patrocinado por el Servicio Nacional de Menores y ejecutado por la Universidad Diego Portales, a partir del cual se estructura el presente artículo.

Esta investigación tiene por objetivo determinar las características y problemáticas que se presentan en el funcionamiento familiar de niños, niñas y adolescentes en situación de graves vulneraciones de derechos, con el fin de generar recomendaciones que permitan guiar la intervención familiar, en pos de la restitución de sus derechos.

\section{Método}

\section{Universo y muestra}

Situando el foco en la relación del niño, niña y adolescente y su familia, se consideró como parte del universo a todo/a niño/a y adolescente vulnerado/a en sus derechos, atendido por algún organismo de la red SENAME (Servicio Nacional de Menores) al momento del estudio, en alguna de las siguientes modalidades de atención: Programas Especializados (explotación sexual comercial infantil, situación de calle y programas integrales especializados), Programas de Protección Especializada en Maltrato y Abuso Sexual, Centros Residenciales de Protección y Programas de Familias de Acogida. A febrero de 2013, el marco muestral a nivel nacional estaba compuesto por 484 centros, los cuales ofertaban un total de 24935 plazas de atención.

\section{Extracción de la muestra}

El sistema de muestreo incorporó diferentes etapas. Como primera fase, se seleccionaron las regiones de las cuales se obtuvo la muestra, utilizando para ello el criterio de mayor concentración de plazas. En cada una de las regiones elegidas, se seleccionaron las capitales regionales y sus conurbaciones donde se concentra la oferta programática. De este modo, la selección de núcleos urbanos fue la siguiente: Provincia de Santiago (Región Metropolitana); Valparaíso-Viña del Mar (V Región); Talca (VII Región) y ConcepciónHualpén (VIII Región).

En la segunda fase, se seleccionaron de modo aleatorio aquellos programas de las líneas programáticas y núcleos urbanos considerados en la muestra. Finalmente, de los centros elegidos se seleccionaron al azar a los sujetos integrantes de la muestra y sus respectivas familias. Dado que el objetivo de este estudio es indagar en la relación de los niños, niñas y adolescentes con sus familias, se utilizó como criterio de inclusión que estos mantuvieran contacto con su familia de origen. En la Tabla 1 , se detalla la distribución de los 34 programas sorteados, distribuidos de modo proporcional por región y línea programática. Del mismo modo, esta tabla da cuenta de la distribución de la muestra final obtenida, la cual asciende a 389 casos. 
Tabla 1

Muestra efectiva final, según región y línea programática

\begin{tabular}{|c|c|c|c|c|c|c|c|c|c|c|}
\hline \multirow{2}{*}{ Región } & \multicolumn{2}{|c|}{ Especializados } & \multicolumn{2}{|c|}{ Maltrato } & \multicolumn{2}{|c|}{ Residencias } & \multicolumn{2}{|c|}{ Familia Acogida } & \multirow{2}{*}{$\begin{array}{c}N \\
\text { centros }\end{array}$} & \multirow{2}{*}{$\begin{array}{c}N \\
\text { muestra }\end{array}$} \\
\hline & Centros & $n$ & Centros & $n$ & Centros & $n$ & Centros & $n$ & & \\
\hline V & 2 & 20 & 2 & 25 & 1 & 23 & 1 & 10 & 6 & 78 \\
\hline RM & 3 & 41 & 5 & 68 & 3 & 25 & 2 & 30 & 13 & 164 \\
\hline VII & 1 & 5 & 1 & 15 & 2 & 23 & 1 & 5 & 5 & 48 \\
\hline VIII & 1 & 15 & 3 & 30 & 5 & 44 & 1 & 10 & 10 & 99 \\
\hline Total & 7 & 81 & 11 & 138 & 11 & 115 & 5 & 55 & 34 & 389 \\
\hline
\end{tabular}

\section{Procedimiento}

Dada la necesidad de resguardar la integridad y el bienestar de los sujetos que forman parte de la muestra, se tomó la decisión de obtener la información del niño/a o adolescente y su familia, a través de una fuente secundaria, en esta oportunidad el equipo a cargo de la intervención en cada caso.

Se seleccionó como instrumento la Escala de Evaluación Familiar de Carolina del Norte (NCFAS-G), la cual permite que los profesionales a cargo del caso puedan evaluar el funcionamiento familiar en los sujetos vulnerados en sus derechos. Este cuenta con evidencias de confiabilidad y validez en Chile (Valencia \& Gómez, 2010); asimismo, la investigación de referencia de Johnson et al. (2008) concluye que esta escala es una de las más relevantes, debido a su enfoque en las fortalezas y a la evidencia acumulada en población vulnerada. Finalmente, un estudio reciente de Kirk (2015), creador de la escala, confirmó los hallazgos positivos de validez y confiabilidad del instrumento.

Para que los/las profesionales de los equipos interventores pudieran utilizar apropiadamente el instrumento anteriormente señalado, recibieron una capacitación de ocho horas por parte de entrenadores certificados, asignándose además un profesional que acompañó en terreno la completación de los instrumentos. Ello permitió resolver las dudas existentes y aunar criterios de puntuación, disminuyendo de este modo la varianza propia de la variable evaluador.

Una vez obtenidos los resultados de la aplicación de este instrumento, se efectuó un análisis estadístico de tipo descriptivo, dando cuenta de las medidas de tendencia central y dispersión. Junto con esto, se llevaron a cabo análisis inferenciales de tipo correlacional.

\section{Resultados}

A continuación, se procede a dar cuenta de los principales resultados del funcionamiento familiar de los niños/as y adolescentes gravemente vulnerados en sus derechos, que integran la muestra. Con fines explicativos, estos resultados han sido organizados de acuerdo a las dimensiones que intervienen en el funcionamiento familiar.

\section{Caracterización del contexto social}

Un $20.8 \%$ de las familias muestra indicadores de alto riesgo en su Entorno, destacando problemas de seguridad en el barrio o vecindario. Un $16.9 \%$ evidencia significativas dificultades de Autonomía, destacando problemas en los ingresos familiares. Un $15.7 \%$ presenta importantes dificultades en el uso de sus redes sociales formales e informales, siendo la conexión con el vecindario y la comunidad el aspecto más deteriorado. En resumen, entre 1 y 2 de cada 10 familias atendidas en la red podrían clasificarse en "alto riesgo socioeconómico", ubicándose la mayoría de los casos en un riesgo social leve.

$\mathrm{Al}$ analizar el contexto social según la línea de intervención, se configura un escenario de complejidad incremental, en que las familias evaluadas en la línea de Familias de Acogida presentan los mejores indicadores, con baja proporción de problemáticas de alta complejidad, seguido de los Programas de Reparación en Maltrato; en el otro extremo, se ubican los Programas Especializados y las Residencias.

Para analizar con mayor detalle las diferencias de perfiles socioeconómicos de las familias según línea programática, se presenta una tabla únicamente con los promedios obtenidos en cada variable evaluada (Tabla 2), los colores rojizos indican las medias más bajas y los colores verdes las variables de mejor desempeño. 


\section{Tabla 2}

Diferencias de promedios en variables de la dimensión Entorno, según línea programática

\begin{tabular}{|l|r|r|r|r|r|}
\hline & $\begin{array}{c}\text { Familia } \\
\text { Acogida }\end{array}$ & $\begin{array}{r}\text { Programas } \\
\text { Reparación }\end{array}$ & $\begin{array}{r}\text { Programas } \\
\text { Especializados }\end{array}$ & Residencias & \multicolumn{1}{c|}{ Total } \\
\hline Entorno general & 0.15 & -0.03 & -0.51 & -0.81 & -0.31 \\
Estabilidad vivienda & 0.65 & 0.46 & -0.01 & -0.07 & 0.24 \\
Seguridad comunidad & 0.13 & -0.25 & -0.62 & -0.75 & -0.41 \\
Riesgos del entorno & 0.25 & 0.31 & -0.35 & -0.39 & -0.02 \\
Habitabilidad de la vivienda & -0.1 & 0.01 & -0.41 & -0.28 & -0.17 \\
Higiene personal & 0.98 & 0.88 & 0.4 & 0.01 & 0.55 \\
Entorno aprendizaje & 0.43 & 0.07 & -0.56 & -0.82 & -0.25 \\
\hline
\end{tabular}

En relación con la Autonomía de la familia (Tabla 3), las temáticas de mayor complejidad son el ingreso familiar y el empleo del cuidador, especialmente en los Programas Especializados. Casi todos los promedios se ubican sobre la línea de base o adecuado en los Programas de Reparación en Maltrato. En los Programas Especializados, además de los temas de empleo, ingreso y manejo financiero, surge como una problemática relevante la comida y nutrición.

\section{Tabla 3}

Diferencias de promedios en variables de la dimensión Autonomía, según línea programática

\begin{tabular}{|l|rrrrrr|}
\hline & $\begin{array}{c}\text { Familia } \\
\text { Acogida }\end{array}$ & $\begin{array}{c}\text { Programas } \\
\text { Reparación }\end{array}$ & $\begin{array}{c}\text { Programas } \\
\text { Especializados }\end{array}$ & Residencias & \multicolumn{1}{c}{ Total } \\
\hline Autonomía general & -0.16 & 0.01 & -0.71 & -0.54 & -0.31 \\
Empleo del cuidador & -0.46 & 0 & -0.67 & -0.51 & -0.34 \\
Ingreso familiar & -0.11 & -0.21 & -0.81 & -0.51 & -0.4 \\
Manejo financiero & 0.2 & 0.05 & -0.56 & -0.33 & -0.15 \\
Comida y nutrición & 0.4 & 0.35 & -0.48 & -0.15 & 0.06 \\
Transporte & 0.55 & 0.49 & -0.04 & 0.26 & 0.33 \\
\hline
\end{tabular}

Respecto a la conexión de la familia con las redes formales e informales, la Tabla 4 muestra que los temas de mayor complejidad incluyen la conexión con el vecindario, seguido por las relaciones sociales con familiares y amigos, aspecto donde aparecen más debilitadas las familias atendidas en Residencias. Por otra parte, es llamativo que las familias muestren, en todas las líneas programáticas, como una fortaleza la iniciativa y aceptación del cuidador respecto a la ayuda ofrecida, constituyéndose en un importante recurso de cara al pronóstico de las intervenciones.

\section{Tabla 4}

Diferencias de promedios en variables de la dimensión Vida Social Comunitaria, según línea programática

\begin{tabular}{|c|c|c|c|c|c|}
\hline & $\begin{array}{l}\text { Familia } \\
\text { Acogida }\end{array}$ & $\begin{array}{l}\text { Programas } \\
\text { Reparación }\end{array}$ & $\begin{array}{l}\text { Programas } \\
\text { Especializado }\end{array}$ & Residencias & Total \\
\hline Vida social/comunitaria en general & 0.22 & -0.32 & -0.55 & -0.72 & -0.4 \\
\hline Relaciones sociales & 0.48 & -0.28 & -0.66 & -0.75 & -0.38 \\
\hline $\begin{array}{l}\text { Relaciones con cuidado infantil, } \\
\text { escuelas }\end{array}$ & 0.55 & 0.12 & -0.51 & -0.73 & -0.17 \\
\hline $\begin{array}{l}\text { Conexión con vecindario y comunidad } \\
\text { cultural/étnica }\end{array}$ & -0.27 & -0.42 & -0.78 & -0.63 & -0.53 \\
\hline $\begin{array}{l}\text { Conexión con la comunidad } \\
\text { espiritual/étnica }\end{array}$ & -0 . & -0.16 & -0.69 & -0.48 & -0.36 \\
\hline $\begin{array}{l}\text { Iniciativa y aceptación del cuidador de } \\
\text { la ayuda y apoyo disponible }\end{array}$ & 0.84 & 0.25 & 0.2 & 0.1 & .28 \\
\hline
\end{tabular}

Caracterización de la dinámica relacional en la familia

En contraste con la menor presencia de factores de riesgo observada en el entorno social, económico y comunitario de las familias atendidas, se observa una proporción mucho mayor de problemáticas de alta complejidad en la dinámica relacional de la familia en general. Así, la siguiente tabla muestra promedios más cercanos a -1 que a 0 .

\section{Tabla 5}

Características generales de la dinámica relacional de las familias

\begin{tabular}{|c|c|c|c|c|}
\hline Dimensión & $\begin{array}{l}\text { Promedio } \\
\text { (DE) }\end{array}$ & $\begin{array}{l}\text { Porcentaje de casos } \\
\text { "alto riesgo" }(-2 \text { o }-3)\end{array}$ & Principal problema & $\begin{array}{c}\text { Promedio } \\
(D E)\end{array}$ \\
\hline Comy & $-0.65(1.37)$ & 31 & Prácticas & \\
\hline terac & $-0.77(1.3)$ & 31.7 & $\begin{array}{l}\text { Relación entre los } \\
\text { propenitores/cuidadores }\end{array}$ & $-0.8(1.53)$ \\
\hline Seguridad familiar & -0.6 & 31 & $\begin{array}{r}\text { progenttores / culdadores } \\
\text { Otros conflictos } \\
\text { familiares }\end{array}$ & $-0.77(1.29)$ \\
\hline Salud familiar & $-0.12(1.21)$ & 13.2 & Salud mental del niño'a & $-0.37(1.29)$ \\
\hline
\end{tabular}

No obstante, es llamativa la alta proporción de casos evaluados con baja complejidad. La línea Familias de Acogida presenta los mejores indicadores, con baja proporción de situaciones de alto riesgo (menos del $10 \%$ para todas las dimensiones), seguido en forma más distanciada por los Programas de Reparación en Maltrato (cercanos al $25 \%$, salvo salud familiar); en el otro extremo, se ubican los Programas Especializados y Residencias de protección (cercanos al $45 \%$, salvo salud familiar) ${ }^{1}$.

La profundización de estos hallazgos según línea programática se detalla a continuación. Como puede observarse en la Tabla 6, los resultados para el área de Competencias Parentales muestran que el tema de mayor complejidad se da en las prácticas disciplinarias, 
lo cual refleja una recurrencia habitual a prácticas de malos tratos referidas como "disciplina", particularmente en familias de Residencias y Programas Especializados ${ }^{2}$. La segunda variable con promedio general más bajo es la literacidad del progenitor, dando cuenta de carencias en la educación del/a cuidador/a o figura parental, dificultad que se observa en todas las líneas evaluadas $^{3}$.

En el caso de las Residencias y de los Programas Especializados surgió también como una importante debilidad la "supervisión del niño/a", y la "entrega de oportunidades de desarrollo y crecimiento". Ambas se asociaron significativamente con otras numerosas variables específicas $^{4}$, a saber: higiene personal del niño; comida y nutrición; entorno de aprendizaje; apego, comunicación y expectativas sobre el niño; apoyo mutuo dentro de la familia; rutinas y rituales familiares; recreación y juego familiar; abuso emocional; negligencia; relaciones sociales y relaciones con el sistema escolar.

Por último, es importante señalar que en las cuatro líneas programáticas se evaluó como nivel adecuado o fortaleza el ámbito de un uso de drogas o alcohol que interfiera con la parentalidad, aspecto que resulta muy marcado en el caso de Familias de Acogida.

Tabla 6

Diferencias de promedios en variables de la dimensión Competencias Parentales, según línea programática

\begin{tabular}{|lrrrrrr|}
\hline & $\begin{array}{c}\text { Familia } \\
\text { Acogida }\end{array}$ & $\begin{array}{c}\text { Programas } \\
\text { Reparación }\end{array}$ & $\begin{array}{c}\text { Programas } \\
\text { Especializados }\end{array}$ & Residencias & Total \\
\hline Competencias parentales general & 0.02 & -0.26 & -1.03 & -1.26 & -0.65 \\
$\quad$ & 0.42 & -0.20 & -1.01 & -1.14 & -0.54 \\
\hline Supervisión del niño & 0.04 & -0.52 & -1.19 & -1.3 & -0.79 \\
$\begin{array}{l}\text { Prácticas disciplinarias } \\
\text { Entrega oportunidades } \\
\text { desarrollo/crecimiento }\end{array}$ & 0.13 & -0.16 & -0.81 & -1.21 & -0.54 \\
$\begin{array}{l}\text { Uso de drogas/alcohol interfiere } \\
\text { parentalidad }\end{array}$ & 1.17 & 1.01 & 0.07 & 0.15 & 0.6 \\
$\begin{array}{l}\text { Estimulacíón parental de la } \\
\text { educación del niño }\end{array}$ & 0.47 & 0.34 & -0.52 & -0.63 & -0.08 \\
$\begin{array}{l}\text { Control acceso a materiales } \\
\text { mediáticos/de lectura }\end{array}$ & 0.26 & 0.17 & -0.85 & -0.79 & -0.28 \\
$\begin{array}{l}\text { Literacidad del } \\
\text { progenitor/cuidador }\end{array}$ & -0.48 & -0.26 & -1 & -0.92 & -0.62 \\
\hline
\end{tabular}

En el área de las Interacciones Familiares, la Tabla 7 muestra que el tópico de mayor complejidad se observa en la relación entre los progenitores/cuidadores ${ }^{5}$. Cabe señalar que el promedio más bajo se obtuvo en los Programas Especializados (-1.28), seguido de las Residencias (-0.96) y los Programas de Reparación en Maltrato (-0.77). La segunda variable con promedio general más bajo es la recreación y juego familiar, siendo la única de las variables específicas del dominio que se ubicó en el rango de problema para las cuatro líneas programáticas $^{6}$.

\section{Tabla 7}

Diferencias de promedios en variables de la dimensión Interacciones Familiares, según línea programática

\begin{tabular}{|lrrrrrr|}
\hline & $\begin{array}{c}\text { Familia } \\
\text { Acogida }\end{array}$ & \multicolumn{1}{c}{$\begin{array}{c}\text { Programas } \\
\text { Reparación }\end{array}$} & $\begin{array}{c}\text { Programas } \\
\text { Especializados }\end{array}$ & Residencias & \multicolumn{1}{c}{ Total } \\
\hline Interacciones familiares general & 0.02 & -0.55 & -1.3 & -1.12 & -0.77 \\
Apego con el niño & 0.55 & -0.08 & -0.95 & -0.86 & -0.38 \\
Comunicación con el niño & 0.33 & -0.49 & -1.19 & -0.94 & -0.64 \\
Expectativas sobre el niño & 0.18 & -0.31 & -1.01 & -0.88 & -0.54 \\
Apoyo mutuo dentro de la familia & 0.44 & -0.2 & -0.79 & -0.9 & -0.42 \\
Relación entre los & 0.25 & -0.77 & -1.28 & -0.96 & -0.8 \\
progenitores/cuidadores & 0.23 & 0.07 & -0.58 & -0.6 & -0.21 \\
Rutinas y rituales familiares & -0.31 & -0.41 & -1.21 & -0.88 & -0.69 \\
Recreación y juego familiar & & & & & \\
\hline
\end{tabular}

En el dominio de Seguridad Familiar (Tabla 8), las variables que muestran mayor deterioro general son otros conflictos familiares, seguida por negligencia parental y abuso emocional. En el caso de la primera de estas variables, resulta la única evaluada como debilidad transversalmente, con el promedio más bajo en los Programas Especializados. Este ítem considera discusiones violentas con vecinos, amenazas recurrentes de exparejas y agresiones con miembros de la familia extensa, entre otros 7

En el caso de negligencia, se observa una media idéntica para los Programas Especializados y las Residencias (-1.16). Ni en Familias de Acogida ni en los Programas de Reparación en Maltrato esta parece ser una temática particularmente relevante, con promedios cercanos al nivel considerado adecuado. Si bien no destaca con el promedio más débil de la dimensión, esta variable es una de la que muestra el mayor número de correlaciones moderado-altas con otras variables de la escala ${ }^{8}$. 


\section{Tabla 8}

Diferencias de promedios en variables de la dimensión Seguridad Familiar, según línea programática

\begin{tabular}{|l|rrrrr|r|}
\hline & $\begin{array}{c}\text { Familia } \\
\text { Acogida }\end{array}$ & $\begin{array}{c}\text { Programas } \\
\text { Reparación }\end{array}$ & $\begin{array}{c}\text { Programas } \\
\text { Especializados }\end{array}$ & Residencias & \multicolumn{1}{c|}{ Total } \\
\hline Seguridad familiar general & 0.18 & -0.48 & -1.19 & -1.01 & -0.67 \\
Violencia doméstica (figuras & 0.44 & -0.2 & -0.93 & -0.44 & -0.34 \\
parentales) & -0.09 & -0.59 & -1.26 & -1.02 & -0.77 \\
Otros conflictos familiares & 0.53 & -0.01 & -0.75 & -0.32 & -0.17 \\
Abuso fisico en el niño & 0.27 & -0.33 & -1.23 & -0.69 & -0.52 \\
Abuso emocional en el niño & 0.87 & -0.24 & -0.91 & -0.2 & -0.22 \\
Abuso sexual en el niño & 0.23 & -0.05 & -1.16 & -1.16 & -0.54 \\
Negligencia en el niño & 0.92 & 0.75 & -0.24 & 0.27 & 0.46 \\
Acceso a armas & & & & & \\
\hline
\end{tabular}

En la dimensión Salud Familiar (Tabla 9), se observa el mayor deterioro en la salud mental del niño, con promedios similares en Programas Especializados, Residencias y Programas de Reparación en Maltrato ${ }^{9}$.

Tabla 9

Diferencias de promedios en variables de la dimensión Salud Familiar, según línea programática

\begin{tabular}{|lrrrrr|r|}
\hline & $\begin{array}{c}\text { Familia } \\
\text { Acogida }\end{array}$ & $\begin{array}{c}\text { Programas } \\
\text { Reparación }\end{array}$ & $\begin{array}{c}\text { Programas } \\
\text { Especializados }\end{array}$ & Residencias & \multicolumn{1}{c|}{ Total } \\
\hline Salud familiar general & 0.37 & 0.09 & -0.59 & -0.33 & -0.12 \\
$\begin{array}{l}\text { Salud fisica del } \\
\text { progenitor/cuidador }\end{array}$ & 0.35 & 0.35 & -0.23 & -0.06 & 0.12 \\
$\begin{array}{l}\text { Invalidez del } \\
\text { progenitor/cuidador }\end{array}$ & 0.92 & 1.29 & 0.81 & 0.82 & 1.02 \\
$\begin{array}{l}\text { Salud mental del } \\
\text { progenitor/cuidador }\end{array}$ & 0.53 & -0.08 & -0.64 & -0.34 & -0.17 \\
Salud fisica del niño & 0.87 & 0.67 & 0.05 & 0.41 & 0.5 \\
Invalidez del niño & 1.35 & 1.36 & 1.18 & 1.22 & 1.29 \\
Salud mental del niño & 0.22 & -0.37 & -0.66 & -0.46 & -0.37 \\
$\begin{array}{l}\text { Acceso familiar a cuidados de } \\
\text { salud/salud mental }\end{array}$ & 0.58 & 0.23 & -0.43 & 0 & 0.08 \\
\hline
\end{tabular}

Bienestar del niño, niña o adolescente

Se evaluó el Bienestar del Niño/a, que considera indicadores de comportamiento, desempeño escolar y relación con otros (hermanos, pares y cuidadores).En términos generales, el $22.6 \%$ de los casos evaluados se ubicó en zona de "alto riesgo" (-2 y -3), con un promedio de -0.55 $(D E=1.29)$ y con el promedio más bajo ubicado en el ítem desempeño escolar, con una media de $-0.56(\mathrm{DE}=1.43)$.

El análisis de promedios por línea programática mostró que el programa con un mayor deterioro en el bienestar de los niños/as y adolescentes se observó en los Programas Especializados y las Residencias, siendo el mejor el de Familias de Acogida (Tabla 10). Las dimensiones con peores indicadores fueron el desempeño escolar, el comportamiento del niño/a y de su relación con la figura parental.

\section{Tabla 10}

Diferencias de promedios en variables de la dimensión Bienestar del Niño, según línea programática

\begin{tabular}{|c|c|c|c|c|c|}
\hline & $\begin{array}{l}\text { Familia } \\
\text { Acogida }\end{array}$ & $\begin{array}{l}\text { Programas } \\
\text { Reparación }\end{array}$ & $\begin{array}{l}\text { Programas } \\
\text { Especializados }\end{array}$ & Residencias & Total \\
\hline Bienestar & 0.26 & -0.23 & -1.18 & -0.95 & -0.55 \\
\hline Comp & 0.13 & & -1.03 & -0.9 & \\
\hline Desempeño escolar & -0.21 & -0.15 & -1.16 & -0.83 & -0.56 \\
\hline $\begin{array}{l}\text { Relación del niño con el } \\
\text { progenitor/cuidador }\end{array}$ & 0.52 & -0.28 & -1.13 & -0.63 & -0.44 \\
\hline $\begin{array}{l}\text { Relación del niño con hermanos } \\
\text { Relación del niño con pares }\end{array}$ & $\begin{array}{l}0.45 \\
0.33\end{array}$ & $\begin{array}{l}0.06 \\
0.08\end{array}$ & $\begin{array}{l}-0.49 \\
-0.81\end{array}$ & $\begin{array}{l}-0.52 \\
-0.41\end{array}$ & $\begin{array}{l}-0.17 \\
-0.21\end{array}$ \\
\hline $\begin{array}{l}\text { Relación del niño con pares } \\
\text { Cooperación/motivación a } \\
\text { permanecer con la familia }\end{array}$ & 1,15 & 0.5 & $\begin{array}{l}-0.81 \\
-0.38\end{array}$ & $\begin{array}{l}-0.41 \\
-0.04\end{array}$ & 0.26 \\
\hline
\end{tabular}

\section{Acumulación de factores de riesgo}

Se evaluó la concentración o acumulación de factores de riesgo severos $(-3)$ en las familias evaluadas por línea programática, encontrándose una diferencia estadísticamente significativa ${ }^{10}$. Así, la presencia de indicadores de riesgo graves se pesquisa en una de cada tres Familias de Acogida, tres de cada cuatro en Programas Especializados, cerca de la mitad de los Programas de Reparación de Maltrato y en dos tercios de Residencias. El $21 \%$ de las familias de Programas Especializados y el $19.6 \%$ de las Residencias se ubicó en la categoría de riesgo extremo, con 10 o más factores de riesgo acumulados, comparado con el 6.8 \% de los Programas de Reparación en Maltrato y el $3.6 \%$ de las Familias de Acogida (Tabla 11).

\section{Tabla 11}

Acumulación de factores de riesgo graves (-3) según línea

\begin{tabular}{|c|c|c|c|c|c|c|}
\hline & & $\begin{array}{c}\text { Programas } \\
\text { Especializados }\end{array}$ & $\begin{array}{l}\text { Programas } \\
\text { Reparación }\end{array}$ & Residencias & $\begin{array}{l}\text { Familias } \\
\text { Acogida }\end{array}$ & Total \\
\hline Sin riesgo & $N(\%)$ & $20(24.7)$ & $76(52.1)$ & $39(36.4)$ & $37(67.3)$ & $172(44.2)$ \\
\hline Bajo riesgo & $N$ & $26(32.1)$ & 4 & $5(23.4)$ & $12(21.8)$ & $104(26.7)$ \\
\hline Moderado riesgo (4 & $N(9$ & 7 & $13(8.9)$ & $13(12.1)$ & $3(5.5)$ & $36(9.3)$ \\
\hline Alto riesgo (7-9) & $N(\%)$ & $11(13.6)$ & $6(4.1)$ & $9(8.4)$ & $1(1.8)$ & $27(6.9)$ \\
\hline Extremo & $N(\%)$ & $17(21)$ & $10(6.8)$ & $21(19.6)$ & $2(3.6)$ & $50(12.9)$ \\
\hline TOTAL & $N(\%)$ & $81(100)$ & $146(100)$ & $107(100)$ & $55(100)$ & $389(100)$ \\
\hline
\end{tabular}

Se observó un promedio de $3.82(\mathrm{DE}=6.8)$ problemas graves (puntuación -3) por familia, con diferencias significativas entre programas $(p=0)$. Las Residencias promediaron 5.75 $(D E=8.9)$, los Programas Especializados 5.5 $(D E=7.1)$, los Programas de Reparación en Maltrato $2.47(D E=5)$ y las Familias de Acogida $1.16(D E=2.6)$. En total, una de cada tres familias se ubicó en una zona de riesgo significativo para el desarrollo infantil (sobre 
3 factores de riesgo). Finalmente, dos de cada cinco familias evaluadas se ubicaron en la zona "sin riesgo" según acumulación de problemáticas graves.

\section{Fortalezas de las familias}

Casi la mitad de las Familias de Acogida muestra fortalezas en su entorno, y más de un tercio muestra fortalezas en su dinámica familiar, con dos de cada cinco niños evaluados positivamente en su bienestar. Prácticamente, un tercio de las familias atendidas en Programas de Reparación en Maltrato se ubican en el rango de fortaleza en todas las dimensiones evaluadas. Esto disminuye notoriamente en los programas especializados y en las Residencias. Si bien se identifican una a dos de cada diez familias con indicadores positivos, casi no se encuentran fortalezas en las interacciones familiares, competencias parentales y bienestar del niño/a. Las mayores fortalezas en Programas Especializados se ubican en el entorno (una de cada cuatro) y en la salud familiar (una de cada cinco). En el caso de las Residencias, las mayores fortalezas están en la autonomía (una de cada cinco) y en la salud familiar (una de cada tres).

\section{Discusión y conclusiones}

Este estudio ha permitido determinar las características centrales del funcionamiento familiar de niños, niñas y adolescentes en situación de graves vulneraciones de derechos y sus principales problemáticas. Entre ellas, se identifica una moderada vulnerabilidad socioeconómica, elevada presencia de dificultades en las interacciones entre sus miembros y con su entorno (familia, vecindario), así como un marcado debilitamiento de las competencias parentales.

El análisis de las evaluaciones realizadas con la escala NCFAS-G, mostró que, en términos generales, el contexto social de las familias atendidas en la red SENAME se caracteriza por problemas leve-moderados en la seguridad del vecindario y la relación entre vecinos. Aunque no se observa un perfil general de extrema pobreza, sí se identifica una vulnerabilidad económica y laboral en los adultos responsables, lo que se asocia a problemas de vivienda y entornos poco estimulantes del desarrollo y aprendizaje de niños/as y adolescentes. Además, se identificó una relativa precariedad en las relaciones sociales con familiares y amigos, lo que redunda en menos recursos de apoyo social disponible, replicando hallazgos de otros investigadores en muestras similares (Rodrigo \& Byrne, 2011).

En cuanto a la dinámica relacional en la familia, destacó la presencia de importantes conflictos entre las figuras parentales en la dinámica cotidiana del hogar, en coherencia con la literatura (Gómez \& Haz, 2008; Gómez, Muñoz, \& Haz, 2007). Estos conflictos parecen irradiar hacia un abanico de procesos del sistema familiar que pueden impactar negativamente en los niños: recreación e interacciones lúdicas escasas, relaciones con redes sociales precarias y débiles, conflictos recurrentes con otras personas externas a la familia.

Las figuras parentales se caracterizan por una baja escolaridad y algunos problemas de salud mental, aunque mucho menos por abuso de drogas y alcohol. Las competencias parentales están debilitadas en los tres pilares principales evaluados por la escala y destacados por Barudy y Dantagnan $(2005,2011)$ : la disciplina parental, la supervisión del niño/a y la promoción del desarrollo y crecimiento mediante oportunidades para el progreso del niño/a o adolescente. Estas dificultades se asocian en forma estadísticamente significativa con las principales vulneraciones identificadas: la negligencia parental y el abuso emocional.

Finalmente, el bienestar del niño/a se observa amenazado principalmente por problemáticas en el desempeño escolar, el comportamiento del niño/a y su relación con la figura parental. Asimismo, se observan indicadores leves de deterioro en la salud mental de los/as niños/as. En una importante proporción de los casos el daño evaluado en la dinámica familiar no se condice con los indicadores de daño en el niño/a, lo que podría dar cuenta de que el deterioro en el funcionamiento familiar en muchas situaciones 
no ha impactado, aun, en forma determinante el desarrollo infanto-juvenil, dando espacio para el trabajo de prevención secundaria.

Cabe señalar que todas estas variables mostraron una graduación de complejidad similar según línea programática, con los indicadores de mayor deterioro en los Programas Especializados y las Residencias, seguidos por los Programas de Reparación en Maltrato y, finalmente, los programas de Familias de Acogida. En este sentido, las familias de acogida muestran, en prácticamente todas las dimensiones, condiciones apropiadas para el desarrollo infantil; familias de acogida que, en la muestra estudiada, están constituidas fundamentalmente por familias extensas que están cumpliendo un rol de acogida. Este resultado constituye un argumento relevante a favor de la apuesta por avanzar decididamente hacia la desinternación de niños y niñas y la transformación del sistema de protección desde grandes instituciones a sistemas de cuidado alternativo de base familiar (Comisión Interamericana de Derechos Humanos [CIDH], 2013).

Es importante destacar que el análisis de factores de riesgo grave mostró que la mayor parte de las familias atendidas en la red SENAME se ubican en la zona de bajo riesgo e incluso que existe un porcentaje no menor de familias que muestran fortalezas en las distintas dimensiones evaluadas. Este hallazgo es relevante, por cuanto estimula a incorporar activamente una perspectiva de resiliencia familiar en la intervención (Gómez \& Kotliarenco, 2010; Walsh, 2004).

El presente estudio permitió identificar una serie de temáticas postergadas, pero prioritarias, por considerar en el diseño de los planes de trabajo y los modelos de intervención. Así, son escasas las experiencias documentadas que intervienen con todo el sistema familiar, ya sea mediante consejerías parentales, consejerías familiares, visitas domiciliarias familiares $\mathrm{O}$ terapia familiar o vincular (Muñoz \& Gómez, 2015; Rodrigo et al., 2015). También escasean las experiencias donde se interviene la pareja como objetivo prioritario de abordaje, ámbito en el cual prácticamente no existe oferta pública.

La evaluación identifica temas centrales que ya son visibilizados en el discurso actual respecto a competencias parentales, pero sin una metodología claramente estructurada y basada en evidencia: disciplina, supervisión y promoción del desarrollo infanto-juvenil. La investigación de las últimas décadas, por ejemplo, habla del valor de apoyar el desarrollo de una disciplina positiva en las familias (Rodrigo et al., 2015). La disciplina parental se asoció significativamente al maltrato (Carreño \& Rey, 2010) y particularmente al abuso emocional, uno de los principales focos de problema evaluados en el área de la seguridad/protección familiar. Así, resulta razonable esperar que mejorando las competencias parentales para el ejercicio de una disciplina positiva, se debería observar menos recurrencia de maltrato emocional y/o físico (Gómez, Cifuentes, \& Ortún, 2012).

La evaluación con la escala NCFAS-G identifica un foco especial en la supervisión del niño, la que mostró una correlación significativa con la negligencia parental, entre otras variables. Mejorando esta competencia, se debería incrementar la restitución de derechos en torno a las vulneraciones asociadas.

La tercera área de intervención prioritaria es la entrega de oportunidades de crecimiento y desarrollo del niño/a o adolescente. En línea con otras publicaciones, esta evaluación aporta argumentos a favor de la propuesta de instalar como un foco prioritario de las intervenciones metodologías que ayuden a las familias a "aprender a jugar", a disfrutar la interacción, en oposición a prácticas que solo enfatizan conceptos como la "responsabilidad", el "deber" o las exigencias. Existe evidencia, desde hace más de 30 años, que el mejor camino para abordar el progreso en la disciplina parental comienza por mejorar la calidad de las interacciones, principalmente introduciendo elementos lúdicos y de disfrute mutuo en la misma (Eyberg et al., 2001; Kaminski, Valle, Filene,\& Boyle, 2008; Rodrigo et al., 2015).

La profundización en el vínculo familiar mostró la importancia de considerar como foco 
técnico de la intervención el fortalecimiento de los buenos tratos, la afectividad y la expresión positiva de emociones en la familia (Barudy \& Dantagnan, 2005; Kaminski et al., 2008; Rodrigo et al., 2015), así como el involucramiento cotidiano y la sensibilidad parental, entendida como la capacidad de leer las señales comunicativas del niño/a, interpretarlas adecuadamente y ofrecer un rango de respuestas sensibles, apropiadas y contingentes (Ainsworth, Blehar, Waters, \& Wall, 1978; Cassidy \& Shaver, 2008). En este sentido, se ha propuesto la sensibilidad parental como la "columna vertebral" de las competencias parentales y la parentalidad positiva (Gómez \& Maureira, 2017).

Asimismo, el estudio identificó como foco relevante de las metodologías de intervención los patrones de comunicación verbal y no verbal entre los miembros de la familia, particularmente en los Programas Especializados y las Residencias de protección.

Finalmente, la evaluación realizada pone de manifiesto que estos núcleos de intervención no deben abordarse sin tomar en cuenta el contexto ecológico en que se despliegan: tan importante como la dinámica relacional al interior de la familia es considerar la autonomía familiar, especialmente en cuanto al empleo estable, y las capacidades laborales y de formación de las figuras parentales (Rodrigo et al., 2015). Asimismo, resulta relevante considerar intervenciones que permitan asegurar un piso mínimo en cuanto a la calidad y estabilidad de la vivienda, enriqueciendo el equipamiento de la misma de tal manera de favorecer el desarrollo y aprendizaje de los niños/as y adolescentes.

En definitiva, este estudio confirma la relevancia de avanzar hacia un modelo ecológico en la intervención realizada en los programas de la red SENAME: aunque el bienestar del niño/ a es prioritario, no es el ámbito más deteriorado. Son las variables de la dinámica relacional en la familia y de pareja, los vínculos afectivos, la parentalidad y la autonomía familiar las que requieren ser transformadas mediante una intervención integral, sistémica, coordinada y oportuna, para garantizar una red protectora de acompañamiento al desarrollo positivo del niño/ a, una "comunidad sensible" con sus integrantes. Los esfuerzos de diseño de metodologías de intervención y los recursos destinados a la capacitación y supervisión técnica de los equipos deberían estar centrados en estos ámbitos en los próximos años, especialmente en los Programas Especializados y las Residencias, donde estos temas resultan más críticos de intervenir.

\section{Agradecimientos}

El estudio que da origen a este artículo fue financiado por la Fundación San Carlos de Maipo y patrocinado por el Servicio Nacional de Menores.

\section{Referencias}

Ainsworth, M., Blehar, M., Waters, E., \& Wall, S. (1978). Patterns of attachment: A psychological study of the strange situation. Hillsdale, NJ: Erlbaum.

Arruabarrena, M. I. (2001). Evaluación y tratamiento familiar. En J. de P. Ochotorena \& M. I. Arruabarrena (Eds.), Manual de protección infantil (2.a ed., pp. 297-340). Barcelona: Masson.

Ayoub, C., O'Connor, E., Rappolt-Schlictmann, G., Vallotton, C., Raikes, H., \& Chazan-Cohen, R. (2009). Cognitive skill performance among young children living in poverty: Risk, change, and the promotive effects of Early Head Start. Early Childhood Research Quarterly, 24, 289-305. https://doi .org/10.1016/j.ecresq.2009.04.001

Barudy, J., \& Dantagnan, M. (2005). Los buenos tratos a la infancia. Barcelona: Gedisa.

Barudy, J., \& Dantagnan, M. (2011). La fiesta mágica y realista de la resiliencia infantil. Barcelona: Gedisa.

Belsky, J. (1993). Etiology of child maltreatment: A developmental-ecological analysis. Psychological Bulletin, 114(3), 413-434. http s://doi.org/10.1037/0033-2909.114.3.413

Belsky, J., Bakermans-Kranenburg, M., \& van IJzendoorn, M. (2007). For better and for worse: Differential susceptibility 
to environmental influences. Current Directions in Psychological Science, 16(6), 300-304. https://doi.org/10.1111/j.1467-87 21.2007.00525.x

Bronfenbrenner, U. (1987). La ecología del desarrollo humano. Barcelona: Piadós.

Bronfenbrenner, U., \& Evans, G. (2000). Developmental science in the 21st century: Emerging models, research designs, and empirical findings. Social Development, 9(1), 115-125. https://doi.org/10.1111/1467-950 7.00114

Bronfenbrenner, U., \& Morris, P. (2006). The bioecological model of human development. En R. M. Lerner (Ed.), Handbook of child development (6.a ed., pp. 793-828). Hoboken, NJ: Wiley.

Canales, P., Flores, M., \& Raurich, C. (2014). Guía de estrategias de intervención familiar. Apoyo para el trabajo con familias en contextos de vulnerabilidad y exclusión social. Santiago de Chile: LOM Ediciones.

Carreño, C., \& Rey, A. (2010). Reflexiones en torno a la comprensión del maltrato infantil. Universitas Psychologica, 9(3), 807-822. Recuperado de http://revistas.javeriana.edu.co/index.p hp/revPsycho/article/view/644/587

Cassidy, J., \& Shaver, P. (2008). Hanbook of attachment. Theory, research, and clinical aplications. Londres: Guildford.

Comisión Interamericana de Derechos Humanos. (2013). El derecho del niño y la niña a la familia. Cuidado alternativo. Poniendo fin a la institucionalización en las Américas (InformeOEA/Ser.L/V/II. Doc. 54/13). Washington: Autor. Recuperado de https://www.oas.org/es/cidh/infancia/do cs/pdf/Informe-derecho-nino-a-familia.pdf

Dubowitz, H.,\& Bennett, S. (2007). Physical abuse and neglect of children. Lancet, 369, 1891-1899. https://doi.org/10.1111/j.13652214.2007.00778_7.x

Evans, G., Li, D., \& Whipple, S. (2013). Cumulative risk and child development. Psychological Bulletin, 139(6), 1342-1396. h ttps://doi.org/10.1037/a0031808
Eyberg, S., Funderburk, B., Hembree-Kigin, T., McNeil, C., Querido, J., \& Hood, K. (2001). Parent-child interaction therapy with behavior problem children: One and two year maintenance of treatment effects in the family. Child and Family Behavior Therapy, 23(4), 1-20. https://doi.org/10.130 0/J019v23n04_01

Gómez, E., Cifuentes, B., \& Ortún, C. (2012). Padres competentes, hijos protegidos: evaluación de resultados del programa "Viviendo en Familia". Psychosocial Intervention, 21 (3), 259-271. ht tps://doi.org/10.5093/in2012a23

Gómez, E., \& Haz, A. M. (2008). Intervención familiar preventiva en programas colaboradores del SENAME: la perspectiva del profesional. Psykhé, 17(2), 53-65. https://doi.org/10.4067/S0718-2228 2008000200005

Gómez, E., \& Kotliarenco, M. A. (2010). Resiliencia familiar: un enfoque de investigación e intervención con familias multiproblemáticas. Revista de Psicología, 19(2), 103-131.Recuperado de http://www. redalyc.org/articulo.oa?id $=26416966005$

Gómez, E., \& Maureira, M. (2017). Manual. Modelo de video-feedback ODISEA 2.0. Oportunidades para el desarrollo de interacciones sensibles, eficaces y afectivas. Santiago: Fundación América por la Infancia.

Gómez, E., Muñoz, M., \& Haz, A.M. (2007). Familias multiproblemáticas y en riesgo social: características e intervención. Psykhé, 16(2), 43-54. https://doi.org/10.406 7/S0718-22282007000200004

Johnson, M., Stone, S., Lou, C., Vu, C. M., Ling, J., Mizrahi, P., \& Austin, M. J. (2008). Family assessment in child welfare services: Instrument comparisons. Journal of Evidence-Based Social Work, 5(1), 57-90. ht tps://doi.org/10.1300/J394v05n01_04

Kaminski, J.W., Valle, L., Filene, J., \& Boyle, C. (2008). A meta-analytic review of components associated with parent training program effectiveness. Journal of Abnormal 
Lorena Contreras Taibo, Catalina Paulsen Gutiérrez, Esteban Gómez Muzzio.

Child Psychology, 36(4), 567-589. https://do i.org/10.1007/s10802-007-9201-9

Kirk, R. (2015). Psychometric properties of the trauma and post-trauma well-being assessment domains of the North Carolina Family Assessment Scale for General and Reunification Services (NCFAS G $+\mathrm{R})$. Journal of Public Child Welfare, 9, 444-462. https://doi.org/10.1080/15548732 .2015 .1090364

Madrid, A. (2015). $4^{\circ}$ Estudio de maltrato infantil en Chile. Análisis comparativo 1994 - 2000. 2006 - 2012. Santiago de Chile: Unicef.

Muñoz, M., \& Gómez, E. (2015). Crecer en familia: un servicio para promover la parentalidad positiva en Chile. En M. J. Rodrigo \& M. L. Máiquez (Eds.), Manual práctico de parentalidad positiva (pp. 189-204).Madrid: Síntesis.

Nemeroff, C. (2016). Paradise lost: The neurobiological and clinical consequences of child abuse and neglect. Neuron, 89(5), 892-909. https://doi.org/10.1016/j.neuron. 2016.01.019

Rodrigo, M. J., \& Byrne, S. (2011). Social support and personal agency in at-risk mothers. Psychosocial Intervention, 20(1), 13-24. http s://doi.org/10.5093/in2011v20n1a2

Rodrigo, M. J., Máiquez, M., \& Martín, J. (2010). Parentalidad positiva y políticas locales de apoyo a las familias. Madrid: Gobierno de España.

Rodrigo, M.J., Máiquez, M., Martín, J., Byrne, S., \& Rodríguez, B. (2015). Manual práctico de parentalidad positiva. Madrid: Síntesis.

Rose, B., Holmbeck, G., Coakley, R., \& Franks, E. (2004). Mediator and moderator effects in developmental and behavioral pediatric research. Developmental and Behavioral Pediatrics, 25(1), 58-67. https://doi.org/10.1 097/00004703-200402000-00013

Valencia, E., \& Gómez, E. (2010). Una Escala de Evaluación Familiar Ecosistémica para programas sociales: confiabilidad y validez de la NCFAS en población de alto riesgo psicosocial. Psykhe, 10(1), 89-103. https://doi.org/10.4067/S0718-222 82010000100007
Walsh, F. (2004). Resiliencia familiar: estrategias para su fortalecimiento. Buenos Aires: Amorrortu.

\section{Notas}

* Artículo de investigación.

1 Estas diferencias fueron estadísticamente significativas en Competencias Parentales, $\operatorname{con} \chi^{2}(6)=55,073 ; p=0$; en Interacciones Familiares, con $\chi^{2}(6)=44,865 ; p=0$; en Seguridad Familiar con $\chi^{2}(6)=41,068 ; p=$ 0 y en Salud Familiar con $\chi^{2}(6)=21,694 ; p$ $=0.001$.

2 El ejercicio de la disciplina parental se asoció significativamente a la presencia de maltrato físico $(r=0.529, p<0.01)$ y emocional $(r=0.639, p<0.01)$, pero también a la existencia de violencia doméstica entre los progenitores/cuidadores $(r=0.557, p<0.01)$. Interesantemente, prácticas disciplinarias es la variable que muestra una asociación más alta de todas en el área de competencias parentales con el ítem general de "bienestar del niño/a" ( $r=$ $0.68, p<0.01)$.

3 La literacidad se asoció significativamente con la estimulación parental de la educación del niño $(r=0.556, p<0.01)$, el control y acceso a materiales mediáticos y de lectura $(r=0.597, p<0.01)$, y la construcción de un entorno de aprendizaje para el niño $(r=$ $0.533, p<0.01$ ).

4 Por la extensión se omitirán los estadísticos, que en todos los casos superan un $r=0.5 \mathrm{y}$ un $p<0.01$

5 Lo cual se asocia significativamente con la presencia de violencia doméstica entre ellos $(r=0.693, p<0.01)$ y a la presencia de otros conflictos familiares $(r=0.543$, $p$ $<0.01$ ). Esta variable mostró la segunda correlación más alta con las dificultades en las rutinas y rituales familiares $(r=0.647, p$ $<0.01$ ), dando cuenta de cómo el conflicto entre las figuras parentales se relaciona con el deterioro general de la dinámica familiar.

6 Esta variable mostró una correlación importante con la organización de un entorno de aprendizaje en el hogar $(r=$ 0.578, $p<0.01$ ), y con las competencias parentales en sus componentes de

| Universitas Psychologica | V. I7 | No.3 | 2018 | 
supervisión $(r=0.624, p<0.01)$, disciplina $(r=0.609, p<0.01)$ y entrega de oportunidades de desarrollo y crecimiento $(r=0.625, p<0.01)$. La asociación más alta con otra variable de la dimensión se observó con la organización de rutinas y rituales familiares $(r=0.727, p<0.01)$.

7 Coherentemente, se observó una asociación significativa de estos conflictos con la variable relaciones sociales $(r=0.5, p$ $<0.01$ ). Las correlaciones más fuertes se identificaron con las rutinas y rituales familiares $(r=0.571, p<0.01)$, relación entre los cuidadores $(r=0.543, p<0.01)$ y apoyo mutuo dentro de la familia $(r=0.543$, $p<0.01)$.

8 Entre estas destaca: abuso emocional $(r=$ $0.662, p<0.01)$, supervisión del niño $(r$ $=0.649, p<0.01)$, estimulación parental de su educación $(r=0.622, p<0.01)$, prácticas disciplinarias $(r=0.621, p<0.01)$, apego $(r=0.62, p<0.01)$, rutinas y rituales familiares $(r=0.613, p<0.01)$ y entrega de oportunidades de desarrollo y crecimiento $(r$ $=0.612, p<0.01$ ), entre otras.

9 Esta variable muestra las asociaciones más altas con el bienestar general del niño $(r$ $=0.524, p<0.01)$, la relación del niño/ a con el progenitor/cuidador $(r=0.507, p$ $<0.01)$, la relación del niño/a con pares $(r$ $=0.507, p<0.01)$, el desempeño escolar $(r=0.47, p<0.01)$ y el comportamiento del niño $(r=0.467, p<0.01)$. La segunda variable con mayor nivel de problemática es la salud mental de las figuras parentales, la que muestra las correlaciones más altas con seguridad familiar en general $(r=$ $0.605, p<0.01)$, interacciones familiares en general $(r=0.598, p<0.01)$ y competencias parentales en general $(r=0.571, p<0.01)$, junto a abuso emocional del niño/a $(r=$ 0.593, $p<0.01)$.

$10 \operatorname{Con} \chi^{2}(12)=45.549 ; p=0$. 